\title{
INTEGRATED SMART SYSTEMS FOR THERANOSTIC APPLICATIONS
}

\author{
Haubold $\mathrm{M}^{1}$, Baum $\mathrm{M}^{1}$, Wiemer $\mathrm{M}^{1}$, Gessner T., \\ ${ }^{1}$ Fraunhofer ENAS, Chemnitz, Germany \\ ${ }^{2}$ Chemnitz University of Technology, Chemnitz, Germany
}

Marco.Haubold@enas.fraunhofer.de

\begin{abstract}
By the incorporation of miniaturised systems within medical devices new functionalities can be achieved and implantable devices profit from a significant increase of functionality. The paper provides information about nowadays and future technical opportunities and focuses on the benefit micro systems can provide to implantable devices and the patients therefore.
\end{abstract}

Keywords: MEMS, Integration, Smart Systems, Medical Device, Packaging

\section{Introduction / state of the art}

MEMS - Micro Electro Mechanical Systems combine the features of measuring physical values and performing the stimulation to their surrounding environment by means of miniaturized mechanical components like springs, functionalized surfaces and optical elements with tiny structures for electrical signal transmission. When combing such sophisticated devices with a digital signal processing unit, adding a communication ability, an energy supply and mechanical anchor points for the fixation at a defined position, a miniaturized smart system is born. The main benefit of those micro machines is to cover most of the tasks of conventional big equipments at a millimetre scale or below with low power consumption and long life time. Within the paper the impact of miniaturized medical devices will be addressed in combination with an overview about the state of the art, current technology developments and future challenges. Finally economical aspects will be considered.

\section{Social impact / Patients point of view}

The impact of the medical device industry on the human life is probably the most direct and beneficial among all high technology industries such as consumer electronics, automobile or space industry. Those products focus on the maintenance and the improvement of our physical condition and therefore effect the basic of our daily life - our health. By the utilization of the advantages miniaturized systems can offer, the variety of addressable application scenarios increase tremendously. This is due to the shrinkage of the component size which features new interactions between the biological and artificial system such as diffusion or tissue conductivity or nonconductivity and provides the opportunity to position the device inside the body, directly at the site of operation without affecting any biological process negatively. Al- ready established examples can be found in hearing aids, where devices are used to actively stimulate parts of the middle ear or the cochlea itself by mechanical mechanisms and sophisticated tiny electrodes. Light sensitive elements are used to be implanted into the human eye at the macula and enable primitive object recognition. Microfluidic systems cope the active or passive directed liquid transport in combination with analysis features either in-vivo or ex-vivo. Further representative micro systems are foreseen to operate inside or adjacent to the human brain performing stimulation (i.e. deep brain stimulation) or measurement tasks like intercranial pressure monitoring systems. Among all the available devices and envisaged research topics, the usability by and the benefit for the patient has seriously to be considered. Beside smart hardware solutions, the addressed systems demand for an intelligent software, filtering the vast amount of data in order to provide a clear statement for the patient and the medical doctor respectively with a certainty and a reliability of 100 percent. As a result, the patient's life quality is enriched by a higher degree of freedom by releasing from stationary treatment and the transition towards health care at home with a "doctor on demand" approach. Hence the recovery from or the life with a crucial decease is improved by the family and the familiar and personal environment of the own home.

\section{Technical challenges and constraints}

The very basic challenge is found at the interface of the particular components to be integrated like detection/actuation element - electronics - communication energy supply (Fig. 1). For the fabrication of those separate parts, new and powerful technologies are utilized (sintering of nano structures, nano lithography, and complex metallization schemes) offering a wide field of possibilities by the application of a vast variety of materials. The result is a complicated interface that is highly sensitive to thermo-mechanical stress due to the mismatch of the applied materials' coefficient of thermal expansion (CTE). Hence a complex process design and material selection is essential. 


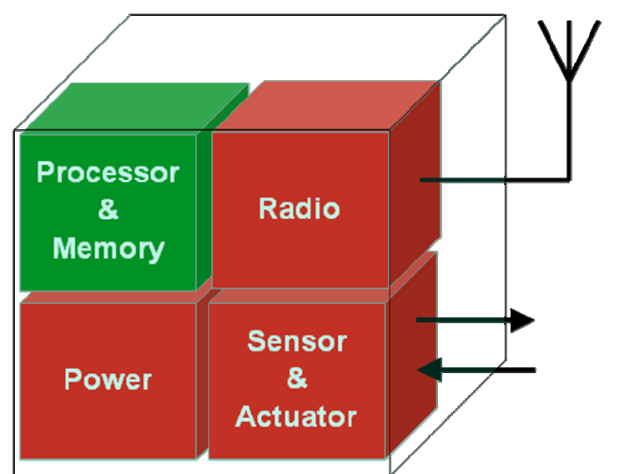

Figure 1: Schematic of a highly integrated smart system [1]

The next packaging level is characterized by the demand on tightness in respect to penetration of fluids and gases into the system, characterized by hermeticity. To provide a hermetic 0-level encapsulation at low process temperatures is an intensively studied research field and several promising technologies have emerged allowing for batch fabrication. The subsequent and outermost layer is the most challenging since it acts as protection layer against degradation by surrounding species, prevents the release of internal materials into the surrounding environment by diffusion but assuring the communication to the external data relay or transponder in contrast. Thin film encapsulation is characterized by a high degree of freedom in means of the design process and is capable of forming multilayer packages for smart systems that suppose to withstand the sterilization process. An integrated three dimensional metallization pattern will cover the demand for a redistribution layer, signal routing and antenna formation for wireless communication. Therefore the site of operation has to be considered since body fluids interact with RF signals significantly. The final technological concern deals with the qualification and testing of the system in respect with national law, what basically loops back to the choice of material and process definition (i.e. DIN EN ISO 10993).

\section{Economical Impact}

Recent analyses concerning the growth of various markets characterize the market for medical devices as promising and show an increasing annual turnover (Fig. 2). This of course is affected by the availability of more different devices for mobile and stationary healthcare, the reduction of production costs per feature and most important the demand by the society where the human body slowly adapts to the people's longer life and requires for technical assistance therefore. In addition, current technology improvements provide a common basic to combine different key technologies that contribute to the medical industry.

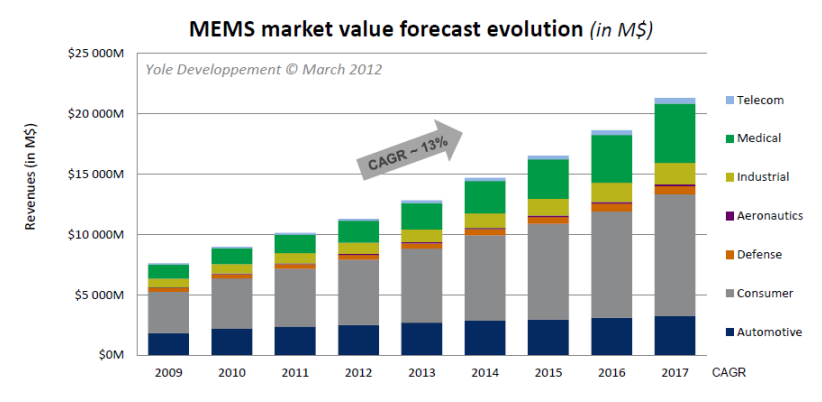

Figure 2: MEMS market forecast predict a market growth for medical devices (green) with a compound annual growth rate of $20 \%$ [2].

\section{Outlook}

Since the research field of miniaturized, smart medical devices is rather new, compared to the established industries, a lot of work has to be done concerning energy supply and storage. Autarkic systems can be achieved by harvesting energy from continuous body processes like vibration, acceleration or temperature gradients [3],[4]. Additionally, the technologies have further to be validated and optimized for producing encapsulation systems that provide bio compatibility and bio stability in combination with the designated conductance behaviour. Within the transmission from a research prototype into a commercially fabricated product, the available financial budget represents the main obstacle, since the qualification and testing in according to national law requires much money and consumes a serious amount of time. So, public funding is a key factor for the success of research projects related to miniaturised medical devices. Beside all the technical assistance systems, the best way to keep in a good physical condition is a balanced life filled with joy and a healthy life style. In the case something unexpected hinders this primary objective, smart systems will have the potential to cope with handicaps and supporting the people during their daily routines.

\section{Bibliography}

[1] Gessner, T. et al.: Smart systems; 10th China Semiconductor Technology International Conference; Shanghai China, 2011, 13-14 March 2011

[2] Yole Dévelopment: MEMS Packaging - Market \& Technology Trend, 2012

[3] Dal Molin, R. et. al.: Novel piezoelectric harvester for cardiac implants: Design, Optimisation \& Fabrication, Proc. Smart Systems Integration, Amsterdam, The Netherlands, 2013, March 13-14

[4] Kim, A. et al.: A novel electromechanical interrogation scheme for implant able passive transponders, IEEE $25^{\text {th }}$ international conference on Micro Electro Mechanical Systems (MEMS), Paris, France, 2012, January 29 February 2 